Volume 9, No.4, July - August 2020

International Journal of Advanced Trends in Computer Science and Engineering

Available Online at http://www.warse.org/IJATCSE/static/pdf/file/ijatcse335942020.pdf

https://doi.org/10.30534/ijatcse/2020/335942020

\title{
Performance Evaluation of Laser Based FSO Communication System in Turbulent Atmosphere
}

\author{
Vijayashri Belgaonkar ${ }^{1}$, R. Sundaraguru ${ }^{2}$ \\ ${ }^{1}$ Department of Electronics \& Communication Engg., Sir MVIT, India, vijayashree_ec@sirmvit.edu \\ ${ }^{2}$ Department of Electronics \& Communication Engg., Sir MVIT, India, sugursg@ sirmvit.edu
}

\begin{abstract}
Laser based Free-space optical communication (FSO) is evolving as one of the key advancements in multi giga byte wireless communication. The turbulence in the atmosphere is the major degrading factor of FSO link. The weak atmospheric turbulent channel capacity of the link has been evaluated using the log normal distribution model. The performance of this model is evaluated based on refractive index turbulent strength parameter, link parameters such as length, operational wavelength of the input signal at the transmitter, receiver aperture and Scintillation Index. The closed form of average capacity of the channel has been derived and the capacity of FSO channel is measured at various turbulence levels. Subcarrier Intensity Modulation/SIM BPSK modulation technique is used in FSO link and error performance is evaluated.SIM BPSK modulation shows better performance as compared to NRZOOK in weak atmospheric turbulence.
\end{abstract}

Key words: Log Normal distribution, Free Space Communication (FSO), refractive index structure parameter, SIM BPSK Modulation.

\section{INTRODUCTION}

Free space optical communication (FSO)or optical wirelesscommunication, has become a well-known research subject in the present business and technical front because of the financial affordability, permission free and high data transmission rates. This rising innovation shows up as a promising expansion to earthly communication applications in contrast to its RF counterparts. Because of its small transmit beam range, the FSO transmission offers LOS connectivity and operates in both visible and IR ranges [1]. The essential rule of FSO employs line-of-sight communication technique.

FSO communication system is preferred over the Radio Frequency counterparts. This is significantly because of the contrast in the transmission bandwidth. For FSO communication the atmospheric transmission range lies at par with the infrared bandwidth run from "700 nm to $1600 \mathrm{~nm}$ ". The transmission range for radio frequency signals lie between $30 \mathrm{~mm}$ to $3 \mathrm{~m}$. This huge proportion of wavelength prompts some intriguing contrasts between them such as huge modulation bandwidth, narrow beam divergence, low power consumption, high data rate, licensefree spectrum and high security. Adding to these advantages, FSO links are readily expandable while eliminating network size, small sized and light weighted. Also, FSO links can be easily and quickly deployed in places where optical fibres cannot be used [2].FSO communication provides a usable bandwidth which is $10^{5}$ times more as compared to the RF counterparts. There is also an increase in the power received at the receiver in case of FSO systems due to the usage of narrower bandwidth. The narrow beam divergence of FSO systems has resulted in small size, light weighted and low power consumption systems. Hence, designers have the flexibility to design smaller systems having higher gain margin at a very affordable initial setup cost and reduced development time. FSO systems also provide license-free transmission and thereby, can be deployed in places where laying of optical fibres is practically not feasible. This also reduces the network size along with ready expandability [3].

FSO technology uses the atmosphere as a proliferating medium whose attributes are influenced by the weather and geographical location of the place, snow, rain, clouds, haze, fog, etc. The difference in the wavelength of the atmospheric particles and that of wavelength used in FSO communication systems leads to scattering phenomenon. These deteriorate the strength of the optical signal and act as a limiting factor for the deployable link distance. This leads to absorption and scattering [4]. The challenge for the FSO communication is turbulent induced fading or scintillation. Scintillation is due to non-homogeneities in the pressure and temperature of the atmosphere along the optical path. Therefore, for the reliable optical path this atmospheric turbulence plays an important role. Turbulence fading can be statistically modelled by "Log normal, Gamma-Gammaand Negative exponential models". Log - Normal model is widely accepted for weak turbulence in the atmosphere [5].

\section{LOG-NORMAL WIRELESS OPTICAL CHANNEL MODEL}

Estimation of the average feasible optical wireless channel capability due to signal amplitude variations and atmospheric turbulence can be calculated and based on the SNR value, atmospheric turbulences ranging from weak to moderate log-normal distribution model can be employed [5]. 
A distribution which is continuous and the value of logarithm of the variable is normally distributed is termed as lognormal channel model. If $\mathrm{Y}$ is the random variable being considered, then $(\mathrm{X})=\exp (\mathrm{Y})$ is a logarithm-normal distribution. Log normal distribution is mainly used for weak turbulent atmosphere [6].

\subsection{Refracrive Index Structure paramater $C_{n}^{2}$}

The Earth's surface when heated up by the sun and mixed with the turbulent winds of the Earth's atmosphere produces drastic variations in the atmosphere called 'optical turbules'. The "refractive index structure parameter" is influenced by the optical laser beam wavelength, pressure of the atmosphere and temperature of the atmosphere. There are drastic variations with respect to the height $h$ from above the ground, the velocity of the wind $v$, time of the day and the geographical location[4].

Various empirical models of $C_{n}^{2}$ has been proposed which include the PAMELA model, Gurvich model, SLC-Day model, Hufnagel-Valley model, Greenwood Model, etc. Out of these, the Hufnagel-Valley model is a very frequently used model for inland regions and daytime viewing conditions due to its dependency on the variation in altitude $h$, wind speed $v$ and near-ground turbulence levels.[7]

The H-V model [5] can be expressed as:

$\mathrm{C}_{\mathrm{n}}^{2}(\mathrm{~h})=\mathrm{C}_{\mathrm{n}}^{2}(0) \exp \left(-\frac{h}{100}\right)+5.94 \times 10^{-53}\left(\frac{v}{27}\right)^{2} \mathrm{~h}^{10} \mathrm{x}$

$\exp \left(-\frac{h}{1000}\right)+2.7 \times 10^{-16} \exp \left(-\frac{h}{1500}\right)$

where, $C_{n}^{2}(0)$ is the value of $C_{n}^{2}$ at the ground level whose value is estimated to be $1.7 \times 10^{-14} \mathrm{~m}^{-2 / 3}$ during daytime and $8.5 \times 10^{-15} \mathrm{~m}^{-2 / 3}$ during the night time. The value of wind speed is also estimated to be around $21 \mathrm{~m} / \mathrm{s}$ in the altitude of $5-20 \mathrm{~km}$ range.

The value of $C_{n}^{2}(0)$ can also be estimated as follows:

$$
\begin{gathered}
C_{n}^{2}(0)=1.29 \times 10^{-12} r_{0}{ }^{-5 / 3} \lambda^{2}-1.61 \times 10^{-13} \theta_{0}^{-5 / 3} \lambda^{2} \\
+3.89 \times 10^{-15}
\end{gathered}
$$

where, $\theta_{0}$ is the isoplanaric angle and $r_{0}$ is the atmospheric coherence length.

The HV-Night model has been modelled for variations in the turbulence conditions at suitable locations in the night and the can be expressed as:

$$
\begin{aligned}
& C_{n}^{2}(h)=1.95 \times 10^{-15} \exp \left(-\frac{h}{100}\right)+8.16 \times 10^{-54} h^{10} \\
& \exp \left(-\frac{h}{1000}\right)+3.2 \times 10^{-17} \exp \left(-\frac{h}{1500}\right)
\end{aligned}
$$

Higher altitude values yield a low value of $C_{n}^{2}$ and the rate of decrease is $h^{-4 / 3}$. For vertiginous links the value of $C_{n}^{2}$ is an integrated value from above sea level to the tip of the atmosphere ranging to about $40 \mathrm{~km}$. In general, the range of variation of $C_{n}^{2}$ is from $" 10^{-17} \mathrm{~m}^{-2 / 3}$ to $10^{-13} \mathrm{~m}^{-2 / 3}$ " for feeble upto strong turbulences. Due to this, there is a difference in the variation of the effect of turbulence between the uplink and downlink communication. The value of $C_{n}^{2}$ is almost assumed to be constant over the horizontal range[8].

\subsection{Average Channel Capacity}

Irregularities in the Earth's atmospheric refractive index values, termed as "optical turbulence". This is responsible for arbitrary changes in the optical signal intensity called 'scintillation' [8]. The changes in the atmospheric temperature and pressure along the optical propagation path results in scintillations eddies of varying capacity and refractive indices resulting in constructive or destructive interferences. These effects increase the bit-error rates at the receiver and result in the degradation of the received signal[9].

An optical wireless channel is an erratically varying channel and the instantaneous random SNR is calculated by $\gamma=$ $s^{2} / N_{0}$. The "average capacity" of a wireless channel $\langle C\rangle$ indicates the optimum rate of accurate transmission. B is the bandwidth of the channel.Hence the average channel capacity can be described as:

$$
\langle C\rangle=\int_{0}^{\infty} \mathrm{B} \log _{2}^{(1+\gamma)} \mathrm{P}_{\gamma}(\gamma)
$$

Scintillations are measured in terms of "scintillation index" $\sigma_{\mathrm{I}}^{2}$, which are dependent on the refractive index structure parameter $C_{n}^{2}$. The expression for $\sigma^{2}$ is given as:

$$
\sigma_{I}^{2}=\frac{\left\langle I^{2}\right\rangle-\langle I\rangle^{2}}{\langle I\rangle^{2}}=\frac{\langle I\rangle^{2}}{\langle I\rangle^{2}}-1
$$

where, $I$ is the irradiance value and $\langle I\rangle$ represents the value of the ensemble.

For weak turbulences, the $\sigma_{I}^{2}<1$, the "Log normal distribution" is used and for strong turbulence $\sigma_{I}^{2} \geq 1$, the field amplitude is Rayleigh distributed. The I-K distribution model is apt for weak to strong turbulences, but due to its difficulty in expressing it in closed form, the GammaGamma distribution is more prominent [10].

For weak turbulent channel models, the Scintillation index in case of plane and spherical waves is expressed as:

- Plane wave

$\sigma_{\mathrm{I}}^{2}=\sigma_{\mathrm{R}}^{2}=1.23 C_{n}^{2} k^{7} L^{11 / 6}$

- Spherical wave

$$
\sigma_{\mathrm{I}}^{2}=0.4 \sigma_{\mathrm{R}}^{2}=0.5 C_{n}^{2} k^{7} L^{11 / 6}
$$

where, $L$ is the FSO link distance, $\sigma_{I}^{2}$ is the Rytov variance which can also be expressed as $\delta^{2}$. $k$ denotes the optical wave number. 
The scintillation index for weak atmospheric turbulence can be expressed as:

$\sigma^{2}=\exp \left[\frac{0.49 \delta^{2}}{\left(1+0.18 \delta^{2}+0.56 \delta^{\frac{12}{5}}\right)^{\frac{5}{6}}}+\frac{0.51 \delta^{2}}{\left(1+0.9 d+0.62 d^{2} \delta^{\frac{12}{5}}\right)^{\frac{5}{6}}}\right]-1$

Where $D=\sqrt{ } \frac{k D^{2}}{4 L}, k=\frac{2 \pi}{\lambda}$ is Optical wave number, $L$ is the link distance between transmitter and receiver, $\lambda$ is operating wavelength of the input signal, $D$ is Receiver's aperture diameter. Considering spherical wave from equation (7) $\delta^{2}$ is described as Rytov variance and is expressed as:

$$
=1.23 C_{n}^{2} k^{7} L^{11 / 6}
$$

Turbulence induced beam scintillation results in temporal fluctuations in the irradiance of the wireless optical beam, and this results in a major signal degradation in the FSO systems[11].The pdf function of log normal with respect to $I$, is given as:

$$
P_{I}(I)=\frac{1}{I \sigma \sqrt{2} \pi} \exp \left(-\frac{\left(\ln (I)+\sigma^{2}\right)^{2}}{2 \sigma^{2}}\right)
$$

Modifying (9) for pdf as a function of $\gamma$ is expressed as:

$$
P_{\gamma}(\gamma)=\frac{1}{2 \gamma \sigma \sqrt{2 \pi}} \exp \left(-\frac{\ln \left(\frac{\gamma}{\mathrm{r}}+\sigma^{2}\right)^{2}}{8 \sigma^{2}}\right)
$$

where, $\Gamma=\frac{\eta E[I]^{2}}{N_{o}}$, Average SNR at the receiverE[.] Expected value of normalized irradiance $I$.Using equation (4) and (10), the average channel capacity for log normal is expressed as:

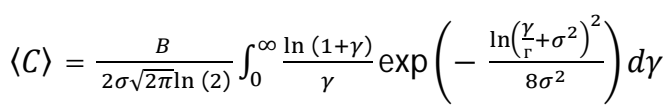

The equation (11) can be transformed into a simplified equation for the estimation of the average capacity of the channel using the mathematical expression "In $(1+x)=$ $\sum_{k=1}^{\infty}(-1)^{k+1} x^{k} / k$ for $0 \leq x \leq 1$ " and the "scaled complementary error function"erfcx $=\exp \left(x^{2}\right) \operatorname{erfc}(x)$, which is a built-in function in MATLAB.

$$
\begin{gathered}
\langle C\rangle=\mathrm{B} \quad C_{0}\left\{\sum _ { k = 1 } ^ { \infty } \frac { ( - 1 ) ^ { k + 1 } } { k } \left[\operatorname{er} f c x\left(\sqrt{2} \sigma k+\frac{A}{2 \sigma \sqrt{2}}\right)+\right.\right. \\
\operatorname{erf} c \mathrm{cx}\left(\left(\sigma k \sqrt{2}-\frac{A}{2 \sigma \sqrt{2}}\right)\right]+\frac{4 \sigma}{\sqrt{2 \pi}}+ \\
\left.\quad A \exp \left(\frac{A^{2}}{8 \sigma^{2}}\right) \operatorname{xer} f c\left(\frac{-A}{2 \sigma \sqrt{2}}\right)\right\}(12)
\end{gathered}
$$

where, $A=\ln (\Gamma)-\sigma^{2}$ and $C_{0}=\exp \left(\frac{-A^{2}}{8 \sigma^{2}}\right) / 2 \ln (2)$

The above equation (12) can be simplified in the following manner:

$$
\begin{gathered}
\langle\hat{\mathrm{C}}\rangle=B C_{0}\left\{\sum_{k=1}^{8} \frac{(-1)^{k+1}}{k} \mid \operatorname{er} f c x\left(\sqrt{2} \sigma k+\frac{A}{2 \sigma \sqrt{2}}\right)+\right. \\
\operatorname{erf} c x\left(\left(\sigma k \sqrt{2}-\frac{A}{2 \sigma \sqrt{2}}\right)\right]+\frac{4 \sigma}{\sqrt{2 \pi}}+ \\
\left.A \exp \left(\frac{A^{2}}{8 \sigma^{2}}\right) \operatorname{xer} f c\left(\frac{-A}{2 \sigma \sqrt{2}}\right)\right\}(14)
\end{gathered}
$$

The above equation (14) provides precise value for the average channel capacity. From equation (11) and (14), we can estimate the error as $(|\langle\hat{C}\rangle-\langle C\rangle| /\langle C\rangle)$. The closed form solution for weak atmospheric turbulent parameter is provided in (14).

\section{BIT-ERROR RATE ANALYSIS FOR WEAK TURBULENCE CHANNEL MODEL}

Presence of noise,hindrances, distortion of the signal due to noise or untimely bit syncing leads toundesirable mismatchin the output bits perceived at the receiver end [12]. BER is a Quality of Service measurement of the system that highlighting the error count in the bits in a stipulated unit of time[13]. Hence BER can gives the likelihood of an erroneous bit being present in the output received bits. This can otherwise mean, that ' 1 ' bit is wrongly perceived as ' 0 ' bit and vice versa. Generally, BER is expressed as:

$$
B E R=P_{0} / 2+P_{1} / 2
$$

where, $P_{0}$ and $P_{1}$ represent the likelihood of wrong detection of ' 0 ' for ' 1 ' and vice versa.

Numerous modulation variations such as the on-off keying (OOK), where the detection of the light pulse in each bit in the train of pulses will result in an "on" and the nondetection will result in an "off" have been used. Since OOK has a very poor energy density and power spectral efficiency, SIM BPSK modulation technique is used. SIM BPSK provides a near-optimum channel capacity. OOK Modulated channel requires need for adaptive threshold therefore subcarrier intensity modulation is preferred. Different data from different users can be accommodated on different subcarriers which increases the capacity of the system. SIM BPSK requires lower bandwidth as compared to PPM.

A single pre-modulated BPSK subcarrier given by $m(t)$ $=g(t) a_{c} \cos (\omega t+\varphi)$, employs a symbol span of $[-1,1]$ for data ' 0 ' and ' 1 '. Coherent demodulation can be employed at the subcarrier level. The coherent demodulator output is given by :

$i_{D}(\mathrm{t})=\frac{R \xi I a_{c} g(t)}{2}[1+\cos (2 \omega t)]+n(t) \cos (\omega t)$

The above-mentioned output, equation (16) when passed through a filter employing low-pass filtering and having a total bandwidth range of $1 / T$, results in the reduction of the noise variance present at the output given by the demodulator. Therefore, there is suppression of the term $\cos (2 \omega t)$ without resulting in distortion to the signal input. Thereby, the received output becomes[13][14], 
$i_{D}(\mathrm{t})=\frac{R \xi I a_{c} g(t)}{2}+n_{D}(\mathrm{t})$

where the term $n_{D}(\mathrm{t})$ denotes the additive noise, whose mean value is zero and variance value is $\sigma^{2} / 2$. It is assumed that both " 0 " and " 1 " is transmitted with equal probability which makes $p_{i}(0)=p_{i}(1)=0.5$.

The probability that the received bits are erroneous given that there is presence of irradiance:

$$
\begin{aligned}
P_{e c}=p_{e}(1) p_{i}(e / 1) & +p_{e}(0) p_{i}(e / 0) \\
= & \frac{2}{4}\left[p_{i}(e / 1)+p_{i}(e / 0)\right](18)
\end{aligned}
$$

The marginal probabilities for both ' 0 ' and ' 1 ' bit transmitted is given by:

$$
\begin{gathered}
p(e / l)=\int_{-\infty}^{0} \frac{1}{\sqrt{\pi \sigma^{2}}} \exp \left\{-\frac{\left(i_{D}(t)-K^{2}{ }^{2}\right.}{\sigma^{2}}\right\} d i_{D}(t) \\
p(e / 0)=\int_{0}^{\infty} \frac{1}{\sqrt{\pi \sigma^{2}}} \exp \left\{-\frac{\left(i_{D}(t)-K^{2}\right.}{\sigma^{2}}\right\} d i_{D}(t)
\end{gathered}
$$

where $\mathrm{K}=\frac{R \xi I}{2}$ and $\mathrm{q}(\mathrm{t})=1$ for $0 \leq \mathrm{t} \leq \mathrm{T}$ and zero otherwise.

Hence BER of thereceived radiance can be be expressed as:

$$
\begin{aligned}
& P_{e}=\int_{0}^{\infty} \frac{1}{\sqrt{\pi \sigma^{2}}} \exp \left\{-\frac{\left(i_{D}(\mathrm{t})-\mathrm{K}^{2}\right.}{\sigma^{2}}\right\} d i_{D}(\mathrm{t}) \\
& P_{e}=1 / 2 \operatorname{erfc}(K / \sigma)=Q\left(\frac{K 2 \sqrt{2}}{2 \sigma}\right)(22)
\end{aligned}
$$

The SNR at the input of the "subcarrier coherent demodulator" is given by:

$\gamma(I)={\frac{(R I \xi)}{\sigma^{2}}}^{2} P_{m}$

where $P_{m}=\frac{A^{2}}{2 T} \int_{0}^{T} g^{2}(t) d t$ and hence $\sqrt{\gamma(I)}=\sqrt{2}(\mathrm{~K} / \sigma)$. The input at the demodulator has an SNR given by :

$$
P_{e}=Q(\sqrt{\gamma(I)})
$$

Using the normalised logarithmic irradiance variation function from (9) to obtain the unconditional probability:

$$
\begin{aligned}
& B E R=\int_{0}^{\infty} P_{e} P_{I}(I) d I \\
& B E R=\int_{0}^{\infty} Q(\gamma(I)) \frac{1}{I \sqrt{2 \pi \sigma^{2}}} \exp \left\{\frac{-\left[\ln \left(I+\sigma^{2}\right]^{2}\right.}{2 \sigma^{2}}\right\} \mathrm{d} I(26)
\end{aligned}
$$

Since a numerical value cannot be computed using the equation (26), a closed form solution can be computed using the Gauss - Hermite quadrature integration approximation, the unconditional BER can be reduced to
$P_{e}=\frac{1}{\sqrt{\pi}} \sum_{i=1}^{n} w_{i} \mathrm{Q}\left(\sqrt{K_{0}} \exp \left(K_{1}\left[\sqrt{2} \sigma x_{i}-\sigma^{2}{ }_{i} / 2\right]\right)\right)$

As noise plays a very important role in the FSO systems, the BER, the values of $K_{1}$ and $K_{0}$ for various noise levels. The Gauss-Hermite function of the order of 20 is used in the above computations.

\section{RESULTS AND DISCUSSIONS}

The results for the weak turbulent atmosphere FSO been simulated in MATLAB and also numerical computations has been carried out for the transfer of data over the channel. We have evaluated the channel capacity the model based on three different weak turbulence strengths $\sigma^{2}$ and refractive index structure parameter $C_{n}^{2}$, as a function dependent on the SNR at the receiver.It is observed that any communication system can be optimised by varying operational wavelength of the signal at the transmitter's end, operational wavelength $\lambda$, the FSO link distance $L$ and the aperture diameter of the receiving system $D$.

The common wavelength used at the transmitter end include $850 \mathrm{~nm}$ and $1550 \mathrm{~nm}$. The closed form equation of $\log$ normal distribution can be used to obtain the graphs for various matrices such as link length, atmospheric turbulence strengths, the structure's refractive index and receiver aperture diameter.

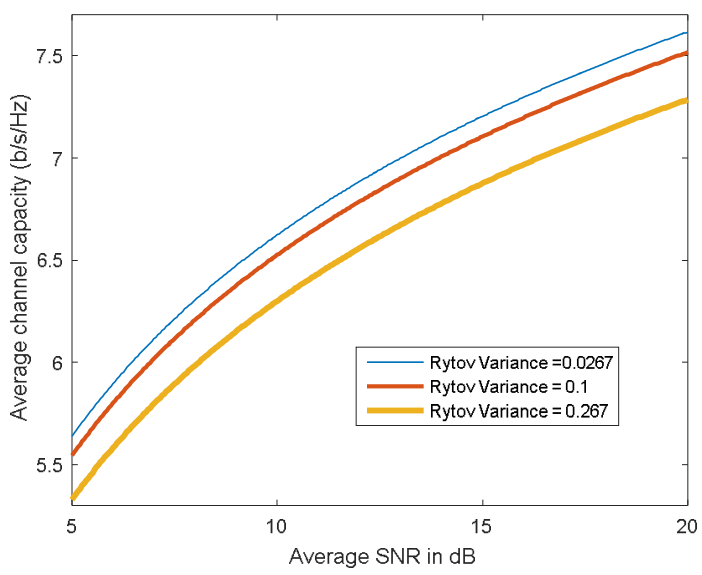

Figure 1:The average channel capacity verses average signal to noise ratio for different rytov variance.

Figure 1 shows "average channel capacity" of log normal distribution at weak turbulent regime. The effect of variation of rytov variance, $\sigma^{2}$ is studied on the plot of Average channel capacity verses SNR in $\mathrm{dB}$ of FSO communication channel employing log-normal distributive model for a link length $\mathrm{L}=1000 \mathrm{~m}, \lambda=1550 \mathrm{~nm}$ and $\mathrm{D}=$ $0.01 \mathrm{~m}$. Rytov variance is the one of the parameter which affects the signal degradation in FSO link. It's clear from the Figure1, as the rytov variance increasesthe channel capacity is degrades. 


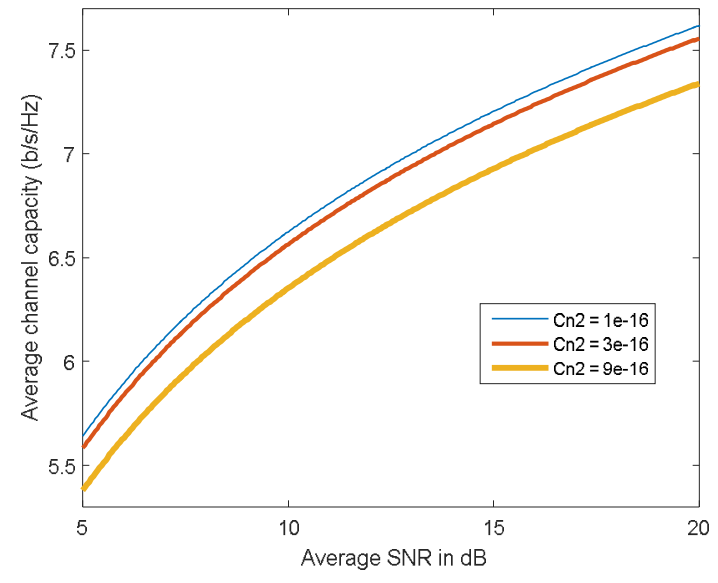

Figure 2:The average channel capacity verses average Signal to noise ratio for different atmospheric turbulence strengths.

Figure 2 shows "average channel capacity" of log normal distribution at weak turbulent regime for different " refractive index structure parameter" $C_{n}^{2}$. The structure parameter depends on the wind speed and height of the communication link from the ground level. As the height from the ground level increases the value of this parameter decreases. In Fig 2 the effect of variation of refractive index structure parameter $C_{n}^{2}$ is observed on the plot of average channel capacity verses SNR in dB of Free Space "Optical Wireless Communication" channel employing "log-normal distribution" for a link length $L=1000 \mathrm{~m}, \lambda=1550 \mathrm{~nm}$ and $D=0.01 \mathrm{~m}$.

From both Figure1 and Figure2, it can be stated that the average channel capacity can be estimated for any value of weak turbulence strength and is strongly influenced by these two parameters. It can be observed that as the value of atmospheric turbulence strength, $\sigma^{2}$ and refractive index structure parameter $C_{n}^{2}$ increases, the capacity of the channel decreases drastically for the given SNR of $20 \mathrm{~dB}$.

The channel capacity is strongly dependent on the distance of the optical communication link and the receiver's "aperture diameter". As the length of the link increases, the sent signal from the transmitter's end should propagate large distances in the atmosphere and hence must bear the influence of the atmospheric turbulences for a longer duration. Link lengths less than $1000 \mathrm{~m}$ are not preferred because the effect of atmospheric turbulence is almost insignificant and hence, lengths $\geq 1000 \mathrm{~m}$.

The effect of atmospheric turbulence can be mitigated using SIM BPSK modulation technique. Figure 3 shows BER verses $\mathrm{SNR}$ in $\mathrm{dB}$ for carious values of atmospheric turbulence. As the turbulence increases the BER also increases as per the plotted results. In this scenario, SIM BPSK Gauss Hermite function is used to evaluate the performance of FSO link for the wavelength of $1550 \mathrm{~nm}$ and link distance of $4000 \mathrm{~m}$.

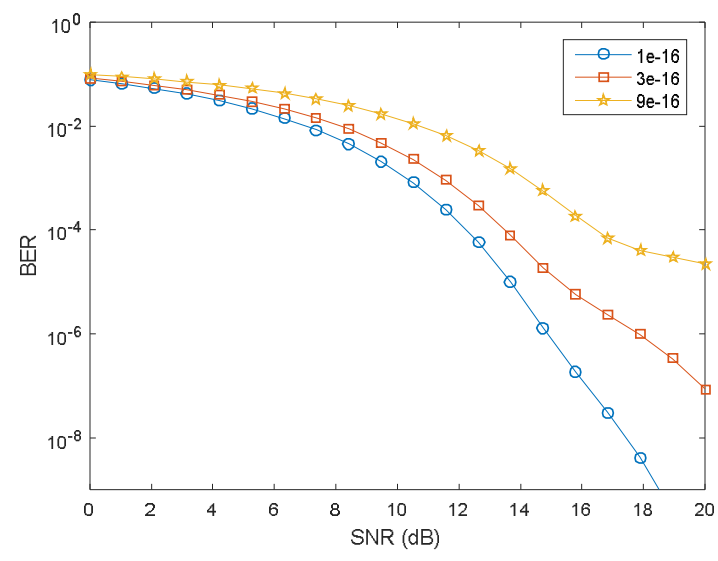

Figure 3: The effect of variation of Refractive index structure parameter $C_{n}^{2}$ by employing SIM BPSK Guass Germite function.

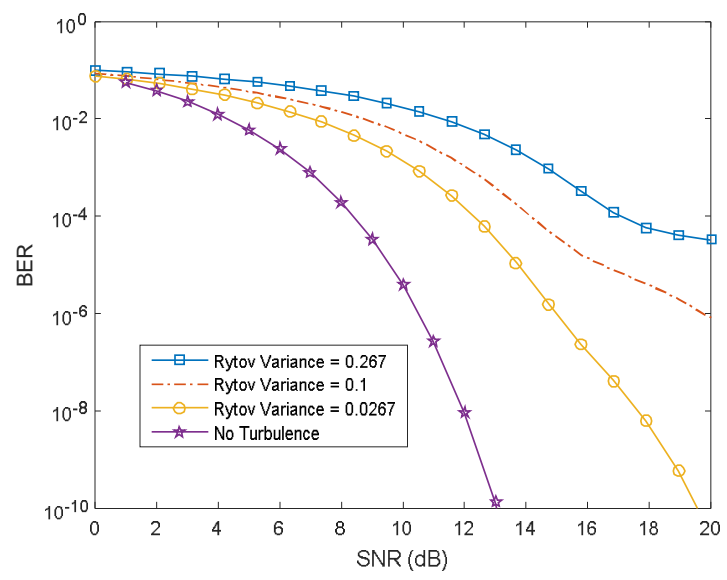

Figure4: The effect of variation of atmospheric turbulence strength $\sigma_{\mathrm{I}}^{2}$, by employing SIM BPSK Guass Hermite function.

Figure 4 shows the BER verses different SNR levels at the receiver using SIM BPSK modulation technique for $\log$ normal distribution. Different values of rytov variances are plotted to observe the effect on FSO link.To plot this, wavelength of FSO link is considered as $1550 \mathrm{~nm}$ and the link range is $4000 \mathrm{~m}$.In Figure 4 plot is also obtained for no turbulence regime which is the ideal case in FSO link. Ideal case is compared with the weak turbulent atmosphere and studied on the plot of average SNR in dB versus the SIM BPSK Hermite Function BER, of a wireless communication channel employing log-normal distribution for $\lambda=1550 \mathrm{~nm}$, $L=4000 m$. 


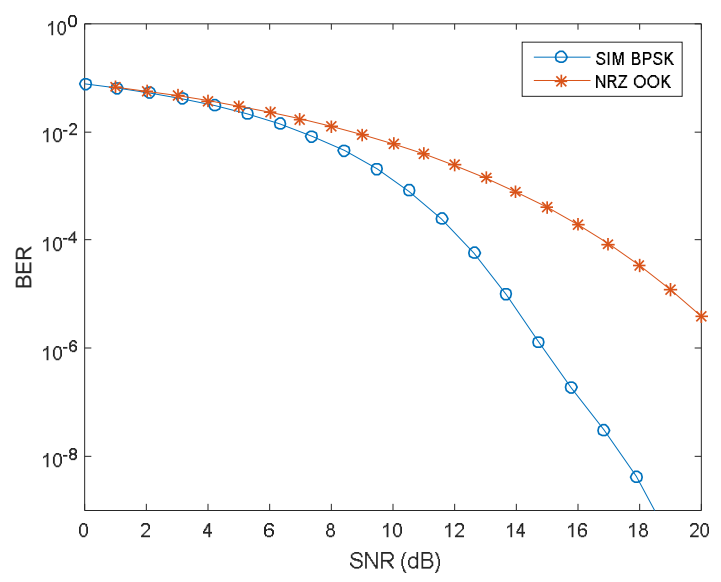

Figure 5: BER verses SNR comparison of SIM BPSK and OOK modulation for the fixed link distance $4000 \mathrm{~m}$ and wavelength $1550 \mathrm{~nm}$.

Figure 5 shows the BER verses different SNR levels at the receiver using SIM BPSK and NRZ OOK modulation techniques for log normal distribution. Its clear from the obtained results that SIM BPSK technique outperforms OOK modulation which is used at many FSO communication links. Bit error rates achieved by SIM BPSK is less compared to OOK. So, from the results obtained for weak turbulence regime SIM BPSK modulation is much suited for FSO link.

\section{CONCLUSION}

In this paper as stated, the average channel capacity for the FSO system is evaluated for the weak turbulent atmospheric channel. The average channel capacity is calculated for various atmospheric turbulence levels like rytov variance and refractive index structure parameter. Wavelength, link ranges and aperture diameters of the receiver have great influence on the capacity of the channel. Adding to this, the SIM BPSK Gauss Hermite function has been used to estimate the BER for different turbulence strengths. It is observed from the obtained results that Gauss Hermite SIM BPSK modulation technique is better suited for the FSO link as compared to NRZ OOK modulation.

\section{REFERENCES}

1. A. K. Majumdar, "Free-space laser communication performance in the ätmospheric channel", J. Opt. Fiber Commun. Rep., vol. 2, no. 4, pp.345-396, Oct. 2005. https://doi.org/10.1007/s10297-005-0054-0

2. M. Khalighi and M. Uysal: Survey on free space optical communication: a communication theory perspective, IEEE Communications Surveys \& Tutorials, vol. 16, no. 4, pp. 2231-2258, Nov. 2014.
3. Yung-Fa Huang, Jong-Shin Chen, Li-Ming Wang, Performance of Transmission Delay and Energy Consumption of Adaptive Listening Mechanism for Wireless Sensor Networks, International Journal of Advanced Trends in Computer Science and Engineering, Vol 8, No.6, Nov-Dec 2019 https://doi.org/10.30534/ijatcse/2019/88862019

4. Hemani Kaushal and georges Kaddoum,Free Space Optical Communication: Challenges and Mitigation Techniques,IEEE Communications Surveys \& Tutorials ( Volume: 19, Issue: 1, Firstquarter 2017 ), pp. 57-96, 2016

5. Hector E. Nistazakis, Evangelia A. Karagianni, Andreas D. Tsigopoulos, Michael E. Fafalios and George S. Tombras, Average Capapcity of Optical Wireless Communication Systems Over Atmospheric Turbulence Channels, Journal Of LightWave Technology, Vol. 27, Issue No. 8, April 15, 2009. https://doi.org/10.30534/ijatcse/2020/116922020

6. A. K. Majumdar and J. C. Ricklin, Free-Space Laser Communications: Principles and Advances, New York,NY,USA,Springer-verlag,Dec. 2010.

7. Z. Ghassemlooy, W. Popoola, and S. Rajbhandari, Optical wireless Communications: System and Channel Modelling with MATLAB, Boca Raton, FL, USA : CRC Press, 2013.

8. Dr.E.Sivanantham, Base Station Positioning in Wireless Networks Using Self-Adaptive Particle Swarm Optimization Approach, International Journal of Advanced Trends in Computer Science and Engineering, Vol 9 No.2, March -April 2020

9. A.C. Boucouvalas, Challenges in Optical Wireless Communications, in Optics \& Photonics News, Vol. 16, No. 5,p.36-39, 2005.

10. E.L Lee and V.W.S. Chan, Optical Communication over the clear atmospheric channel using diversity, IEEE J. Sel. Areas Communications, Vol. 22, No. 9, p.1896-1906, Nov. 2004.

11. M.A. Al-Habash, L. C. Andrews amd R. L. phillips, Mathematical model for the irradiance PDF of a laser beam propagating through turbulent media, Opt.. Eng., Vol. 40, No. 8, pp.1554-1562, 2001.

https://doi.org/10.1117/1.1386641

12. C.C. Davis, I.I. Smolyaninov, Effect of atmospheric turbulence on bit-error rate in an On-Off-keyed optical wireless system, in Free Space Laser.

13. George D. Papadopoulos, George K. Karagiannidis, Fotini-Niovi Pavlidou,Performance blunds of spacetime block coding in Rician and log-normal fading channels, IET Communications, 1, March 2007, pp.8691.

14. Laourine, A. Stephenne and S. Affes, Capacity of lognormal fading channels, in Proc. IWCMC, 2007. 